\title{
Performance of resting metabolic rate estimation equations in obese patients
}

\author{
Milene Moehlecke*, Manoel Roberto Maciel Trindade, Ana Carolina Mazzuca, Carina Andriatta Blume, \\ Jakeline Rheinheimer, Cristiane Bauermann Leitão \\ From 20th Brazilian Diabetes Society Congress \\ Porto Alegre, Brazil. 11-18 November 2015
}

\section{Background}

Weight gain may be associated with an imbalance between energy intake and energy expenditure. The resting metabolic rate (RMR) is the main component of total energy expenditure, and is related mainly to lean mass (LM), as well as to other factors such as fat mass (FM), age, sex and genetic factors. A RMR lower than expected may be a risk factor for weight gain. RMR is estimated by equations that use patient's weight, sex, age and height to calculate energy needs. Several studies have shown that these equations have a poor agreement with RMR measured by indirect calorimetry (IC) in obese patients once their excess fat-free mass (FFM) is usually not taken into account.

\section{Objective}

To evaluate the accuracy of five equations in predicting RMR in obese subjects. Results were compared with measured RMR (mRMR) determined by IC.

\section{Materials and methods}

Cross-sectional study was conducted in obese Southern Brazilian volunteers recruited from community. Body mass index (BMI) was calculated by dividing weight (in kilograms) by squared height (in meters). Body composition was evaluated by dual-energy X-ray. RMR was measured by IC (Weir equation) and estimated (eRMR) by Mifflin-St. Jeor, Owen, Harris-Benedict, Ireton-Jones and Horie-Waitzberg \& Gonzalez (H \& WG) equations (Figure 1). The latter takes into consideration the FFM. Equations performance were determined by bias (mean difference between mRMR and eRMR); precision (standard deviation of bias) and by accuracy (percentage of estimates within $5 \%$ of $\mathrm{mRMR}$ ).

\section{Results}

Sixty individuals (46 women [75\%], 48 white [84\%]) aged $46 \pm 13$ yrs. (range, $21-83$ yrs.) were evaluated. Overall, mRMR was $1941 \pm 642 \mathrm{kcal} /$ day. mRMR increased along

\begin{tabular}{|c|c|}
\hline \multirow{2}{*}{ Harris-Benedict } & Male: RMR $=66.47+1375 \times 5 \mathrm{~W}+50 \times \mathrm{H}-6.75 \times \mathrm{A}$ \\
\hline & Female: $\mathrm{RMR}=65509+9.56 \times \mathrm{BW}+1.84 \times \mathrm{H}-4.67 \times \mathrm{A}$ \\
\hline \multirow{2}{*}{ Owen } & Male: $\mathrm{RMR}=879+10.2 \times \mathrm{BW}$ \\
\hline & Female: $\mathrm{RMR}=795+7.18 \times \mathrm{BW}$ \\
\hline Ireton-Jones & $R M R=629-(11 \times A)+(25 \times B W)-609$ \\
\hline \multirow{2}{*}{ Miflin St Jeor } & Male: $9.99 \times 8 \mathrm{~W}+6.25 \times \mathrm{H}-4.92 \times A+5$ \\
\hline & Female: RMR $=9.99 \times B W+6.25 \times \mathrm{H}-492 \times \mathrm{A}-161$ \\
\hline$H W \& G$ & $R M R=560.43+(5.39 \times B W)+(14.14 \times F F M)$ \\
\hline
\end{tabular}

Figure 1 Prediction equations for comparison with indirect calometry in obese subjects.

\footnotetext{
*Correspondence: milenemoehlecke@yahoo.com.br UFRGS, Porto Alegre, Brazil 


\begin{tabular}{|c|c|c|c|c|c|}
\hline & \multirow{2}{*}{$\begin{array}{c}\text { All } \\
\text { patients }\end{array}$} & \multicolumn{3}{|c|}{ BMI $\left(\mathbf{k g} / \mathrm{m}^{2}\right)$} & \multirow{2}{*}{$\mathbf{P}$} \\
\hline & & $30-34.9(n=21)$ & $36-39.9(n=9)$ & $\geq 40$ (n=30) & \\
\hline Age (years) & $46 \pm 13$ & $46 \pm 14$ & $48 \pm 11$ & $43 \pm 11$ & 0.093 \\
\hline White (\%) & $53(84 \%)$ & $17(80 \%)$ & $7(77 \%)$ & $26(89 \%)$ & 0.710 \\
\hline Women $(\%)$ & $49(77 \%)$ & $16(76 \%)$ & $5(55 \%)$ & $25(83 \%)$ & 0.394 \\
\hline $\begin{array}{l}\text { Actual weight } \\
\text { (kg) }\end{array}$ & $104 \pm 27$ & $83 \pm 10$ & $99 \pm 17$ & $126 \pm 19$ & $<0.001$ \\
\hline LM (kg) & $52 \pm 12$ & $46 \pm 11$ & $55 \pm 11$ & $58 \pm 9$ & $<0.001$ \\
\hline FM $(\%)$ & $44 \pm 7$ & $41 \pm 4$ & $41 \pm 9$ & $50 \pm 5$ & $<0.001$ \\
\hline mRMR (kcal/day) & $1941 \pm 642$ & $1687 \pm 481$ & $2040 \pm 724$ & $2236 \pm 603$ & $<0.001$ \\
\hline
\end{tabular}

Figure 2 Obese patients distributed by anthropometric and body composition parameters.

\begin{tabular}{|c|c|c|c|c|c|}
\hline Equation & $\begin{array}{c}\text { RMR } \\
\text { (kcal/day) }\end{array}$ & $\begin{array}{l}\text { Bias (kcallday) and } \\
\text { limits of agreement } \\
\text { ( } \pm 2 \text { s.d.; kcal/day) }\end{array}$ & Precision & $P$ value & $P_{5}(\%)$ \\
\hline mRMR & 1941 & & & & \\
\hline $\begin{array}{l}\text { Harris- } \\
\text { Benedict }\end{array}$ & 1801 & $148(13 ; 282)$ & 467 & 0.032 & 68 \\
\hline Ireton Jones & 2241 & $-199(-388 ;-9)$ & 681 & 0.040 & 47 \\
\hline Owen & 1642 & $299(154 ; 443)$ & 549 & $<0.001$ & 55 \\
\hline Mifflin & 1709 & $245(107 ; 381)$ & 521 & 0.001 & 60 \\
\hline HW \& G & 1957 & $-17(-148 ; 115)$ & 503 & 0.801 & 70 \\
\hline
\end{tabular}

Figure 3 Comparison between estimated RMR from several equations and measured RMR in obese patients.

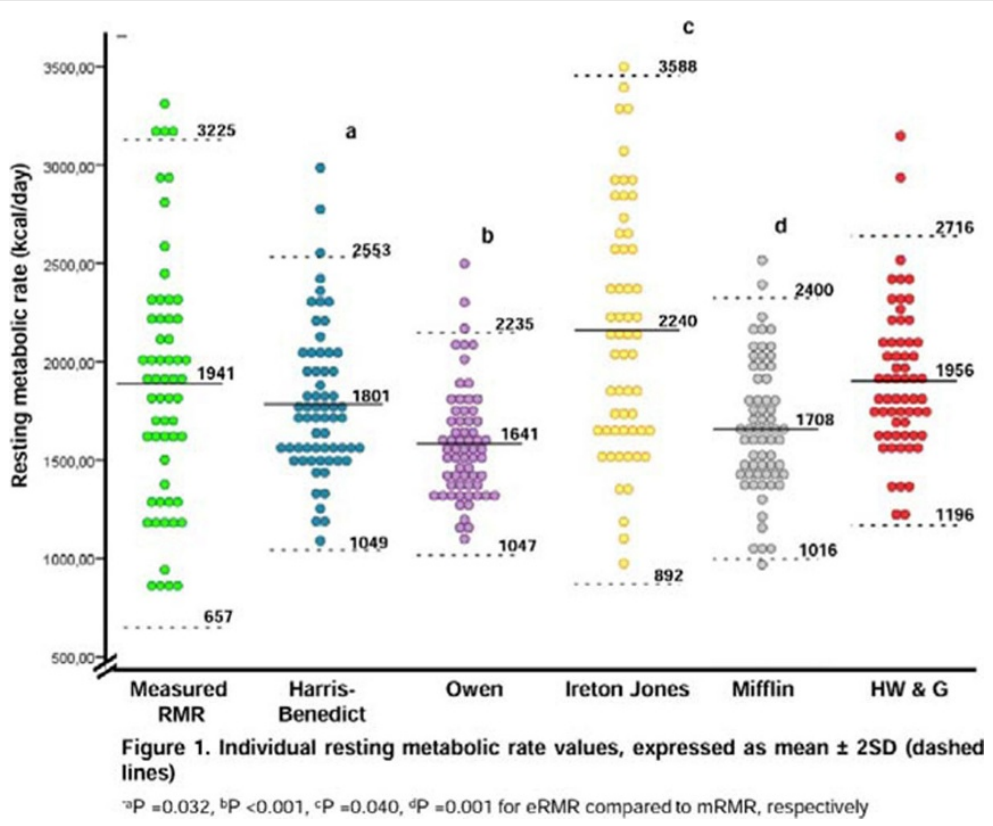

Figure 4 Individual resting metabolic rate values, expressed as mean \pm 2SD (dashed lines) 
with BMI (Figure 2), but the association was lost when corrected for LM $(\mathrm{P}=0,859)$. $\mathrm{H}$ \& WG equation was the only equation unbiased $(\mathrm{P}=0.801)$ (Figure 3$)$. The HarrisBenedict, Owen and Mifflin-St. Jeor equations were biased overall toward underestimation, while Ireton-Jones equation was biased toward overestimation (Figure 4). Bias was significantly higher in women for Harris-Benedict, Mifflin St. Jeor and Owen equations. Accuracy to estimate RMR at $\pm 5 \%$ was suboptimal for all equations, except for H \& WG.

\section{Conclusion}

In this sample of obese subjects, the available RMR estimate equations that do not take into account the FFM have poor accuracy when compared with mRMR.

Published: 11 November 2015

doi:10.1186/1758-5996-7-S1-A231

Cite this article as: Moehlecke et al:: Performance of resting metabolic rate estimation equations in obese patients. Diabetology \& Metabolic Syndrome 2015 7(Suppl 1):A231.
Submit your next manuscript to BioMed Central and take full advantage of:

- Convenient online submission

- Thorough peer review

- No space constraints or color figure charges

- Immediate publication on acceptance

- Inclusion in PubMed, CAS, Scopus and Google Scholar

- Research which is freely available for redistribution

Submit your manuscript at www.biomedcentral.com/submit 